\title{
Rate coefficients from quantum and quasi-classical cumulative reaction probabilities for the $S\left({ }^{1} D\right)+H_{2}$ reaction
}

\author{
P. G. Jambrina, ${ }^{1}$ Manuel Lara, ${ }^{2, a)}$ M. Menéndez, ${ }^{1}$ J.-M. Launay, ${ }^{3}$ and F. J. Aoiz ${ }^{1, b)}$ \\ ${ }^{1}$ Departamento de Química Física, Facultad de Química, Universidad Complutense, 28040 Madrid, Spain \\ ${ }^{2}$ Departamento de Química Física Aplicada, Facultad de Ciencias, Universidad Autónoma de Madrid, \\ 28039 Madrid, Spain \\ ${ }^{3}$ Institut de Physique de Rennes, UMR CNRS 6251, Université de Rennes I, F-35042 Rennes, France
}

(Received 14 August 2012; accepted 8 October 2012; published online 31 October 2012)

\begin{abstract}
Cumulative reaction probabilities (CRPs) at various total angular momenta have been calculated for the barrierless reaction $\mathrm{S}\left({ }^{1} D\right)+\mathrm{H}_{2} \rightarrow \mathrm{SH}+\mathrm{H}$ at total energies up to $1.2 \mathrm{eV}$ using three different theoretical approaches: time-independent quantum mechanics $(\mathrm{QM})$, quasiclassical trajectories (QCT), and statistical quasiclassical trajectories (SQCT). The calculations have been carried out on the widely used potential energy surface (PES) by Ho et al. [J. Chem. Phys. 116, 4124 (2002)] as well as on the recent PES developed by Song et al. [J. Phys. Chem. A 113, 9213 (2009)]. The results show that the differences between these two PES are relatively minor and mostly related to the different topologies of the well. In addition, the agreement between the three theoretical methodologies is good, even for the highest total angular momenta and energies. In particular, the good accordance between the CRPs obtained with dynamical methods (QM and QCT) and the statistical model (SQCT) indicates that the reaction can be considered statistical in the whole range of energies in contrast with the findings for other prototypical barrierless reactions. In addition, total CRPs and rate coefficients in the range of $20-1000 \mathrm{~K}$ have been calculated using the QCT and SQCT methods and have been found somewhat smaller than the experimental total removal rates of S $\left({ }^{1} D\right)$. ( 2012 American Institute of Physics. [http://dx.doi.org/10.1063/1.4761894]
\end{abstract}

\section{INTRODUCTION}

The cumulative reaction probability (CRP) function was originally introduced by Miller ${ }^{1}$ in the context of a quantum mechanical (QM) and semiclassical version of the transition state theory (TST) and it was subsequently developed in a series of articles. ${ }^{2-4}$ The CRP may be interpreted qualitatively as the effective number of reactant states that can lead to the formation of products at a given total energy, E. Actually, the calculation of the CRP does not necessarily involve any approximation and provides a powerful conceptual link between the formalism of the (exact) scattering theory and the simpler and perhaps more intuitive albeit approximate TST. The CRP formalism serves to calculate directly specific and canonical rate coefficients. However, even though the CRP is an averaged property, its dependence on energy contains valuable information about the quantized spectrum of the transition state and the detailed dynamics of the reaction. Indeed, the analysis of the CRPs has allowed the identification of these quantized transition states or reaction bottlenecks ${ }^{5-7}$ that also seem to manifest in state-to-state descriptions of the reaction. ${ }^{8}$

At the beginning, TST was conceived to determine thermal rate coefficients for direct reactions using information restricted to the saddle point of the PES. ${ }^{9}, 10$ In 1970s, motivated by the experimental studies on barrierless reactions, ${ }^{11,12}$ Miller extended the TST model to determine rate coefficients for situations in which the definition of the saddle point was

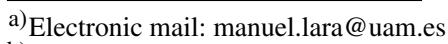

b) Electronic mail: aoiz@quim.ucm.es.
}

not so straightforward. ${ }^{2}$ As an instance, for reactions with deep potential wells, the unified-TST rationalizes the dynamics in terms of two dynamical bottlenecks or dividing surfaces: one before and another after the well, near the saddle points of the possible entrance and exit barriers. In the absence of dynamical barriers, the bottlenecks correspond to the maxima of the centrifugal barrier for each arrangement channel. If the barriers, either dynamical or centrifugal, disappear the dividing surfaces shift to the asymptotic configurations of reactants and products.

Determination of exact QM CRPs covering the whole range of total angular momenta is usually very demanding computationally because the calculation of the full scattering $\boldsymbol{S}$-matrix is required, comprising all total angular momenta, $J$, necessary for convergence. However, more efficient approaches, such as the flux-flux autocorrelation method, allow a direct calculation of thermal-CRPs ${ }^{4}$ circumventing the calculation of the full $S$-matrix. In addition, a quasiclassical trajectory (QCT) version of the CRP formalism has been recently developed ${ }^{13}$ not only for zero total angular momentum ${ }^{14,15}$ but for $J \geq 0$, as well as for the calculation of the total CRP. This method has been applied to the study of a variety of three atom reactions, ${ }^{13,16-18}$ with a generally good agreement with the exact QM calculations. In particular, the QCT CRP method has been employed in the study of the $\mathrm{H}^{+}+\mathrm{H}_{2}$ reaction, ${ }^{18}$ the prototypical barrierless reaction governed by a deep well. In addition, the statistical QCT method (SQCT) ${ }^{19,20}$ has been also used to determine the CRPs and rate coefficients for the $\mathrm{H}^{+}+\mathrm{D}_{2}$ and other isotopic variants. ${ }^{18,21}$ The results obtained for those reactions 
showed that the dynamical methods (QM and QCT) and the statistical model were in good agreement at low energies and low total angular momenta. However, with increasing $J$, it becomes clear that the statistical approach overestimates the QM and, especially, the QCT reactivity. ${ }^{22,23}$ This discrepancy was attributed to the predominance of a direct mechanism for large $J$ values at which the centrifugal barrier acts as a genuine barrier and practically overrides the effect of the well.

The $\mathrm{S}\left({ }^{1} D\right)+\mathrm{H}_{2}\left(\mathrm{X}^{1} \Sigma_{g}^{+}\right) \rightarrow \mathrm{SH}+\mathrm{H}$ reaction has been widely studied, both experimentally $24-27$ and theoretically. ${ }^{28-41}$ Given the vast amount of available information, the system has become a benchmark for insertion, barrierless reactions. ${ }^{28,42}$ It has been also found that, at least at low energies, the reaction takes place mostly via an insertion mechanism involving a long-lived complex. Recent experiments at very low collision energies performed by Costes co-workers ${ }^{43}$ and Sims and co-workers ${ }^{44}$ as well as the new set of PESs calculated by Song et al., ${ }^{46,47}$ as an alternative to the widely used reduced-kernel Hilbert space (RKHS) PES by Ho et al. ${ }^{30}$ have attracted a renewed interest on this system. In this paper, we will apply the methodology inherent to the CRP formalism to the $\mathrm{S}\left({ }^{1} D\right)+\mathrm{H}_{2}$ reaction. This reaction is in some aspects akin to the $\mathrm{H}^{+}+\mathrm{H}_{2}$ family of reactions, dominated by a deep potential well $(\sim 4 \mathrm{eV})$ but with a different mass combination, more prone to produce a long lived complex ${ }^{48}$ irrespective of the collision energy or the total angular momentum.

The aim of this work is twofold. On the one hand, we will describe the adiabatic dynamics for this reaction in terms of CRPs that can be directly used to obtain thermal rate coefficients. On the other hand, the results of calculations carried out on the RKHS PES ${ }^{30}$ and the double-many-bodyexpansion with scaled external correlation (DMBE/SEC) PES $^{47}$ will be compared. The application of three different dynamical methodologies, time-independent QM, QCT, and SQCT is intended to assess the importance of quantum and non-statistical effects for this reaction. As for the comparison of different PESs, recent studies ${ }^{49,50}$ have shown that the $\mathrm{DMBE} / \mathrm{CBS}^{46}$ is inaccurate and incapable to describe correctly the reactivity at small collision energies due to the presence of spurious features in the PES. The DMBE/SEC PES, ${ }^{47}$ however, does not present artifacts and its agreement with the RKHS PES is considerably better. ${ }^{50}$

The paper is organized as follows. Details of the calculations as well as a brief summary of the various theoretical methods are described in Sec. II. The results and its discussion are given in Sec. III. Finally, Sec. IV is devoted to the conclusions of this work.

\section{THEORETICAL METHODS AND CALCULATION DETAILS}

\section{A. Quasi-classical trajectory calculations}

The quasi-classical trajectory method to calculate the cumulative reaction probabilities has been described previously ${ }^{13,16,18,51}$ and only some details will be given here. It must be recalled that the total angular momentum, $J$, rota- tional angular momentum, $j$, and the helicity, $\Omega$, are quantized at the beginning of the trajectory.

CRPs were obtained for $J=0,4,10,30$, and 50 on the RKHS PES ${ }^{30}$ as well as for $J=0$ on the DMBE/SEC PES ${ }^{47}$ by running batches of $5 \times 10^{5}$ trajectories in the total energy range up to $1.5 \mathrm{eV}$. Trajectories were started at an initial distance of $10 \AA$ and the integration step was set to $0.01 \mathrm{fs}$ (on the RKHS PES) and 0.06 fs (on the DMBE/CBS PES), sufficiently small to ensure an energy conservation better than 1 in $5 \times 10^{5}$.

Total CRPs as a function of the total energy were also calculated on both PES as in Ref. 18 by running batches of $5 \times 10^{6}$ trajectories in the same total energy range. Extra batches of $10^{6}$ trajectories were run in the low collision energy range between $10^{-4}-0.1 \mathrm{eV}$, the trajectories starting at $30 \AA$ due to the importance of the long range interactions at those energies.

Once the total CRP has been obtained by using the methodology described above, the expression of the thermal rate coefficient for a homonuclear molecule $\mathrm{B}_{2}$, assuming a thermal equilibrium distribution of internal states, can be written as ${ }^{13}$

$$
k(T)=\frac{\int_{0}^{\infty} C_{r}(E) \exp \left(-E / k_{B} T\right) d E}{h \Phi_{\mathrm{rel}}(T) Q_{v, j}^{\mathrm{B}_{2}}(T)},
$$

where the total CRP as a function of the total energy, $E$, is given by

$$
C_{r}(E)=\sum_{\mathrm{T}}(2 \mathrm{~T}+1) \sum_{J=0}^{J_{\max }}(2 J+1) C_{r}^{J, \mathrm{~T}}(E)
$$

and $\mathrm{T}$ is the molecular nuclear spin quantum number. For the $\mathrm{H}_{2}$ molecule, where $I=1 / 2, \mathrm{~T}=0$ for para-hydrogen (even rotational states) and $\mathrm{T}=1$ for ortho-hydrogen (odd rotational states). The CRP for a given value of the total angular momentum and diatomic parity is $C_{r}^{J, \mathrm{~T}}(E)$. In Eq. (1) $k_{B}$ is the Boltzmann constant, and $\Phi_{\text {rel }}(T)$ and $Q_{v, j}^{\mathrm{B}_{2}}(T)$ are the relative translational and coupled nuclear-rovibrational partition functions, respectively.

Equation (1) together with Eq. (2) assume that there is ortho-para equilibrium at any temperature. However, in the experiments carried out to determine the $S\left({ }^{1} D\right)$ removal rate coefficients at low temperatures, the paralortho fraction is that corresponding at room temperature $(\approx 1: 3)$. The underlying hypothesis is that, during the expansion (and previous cooling) to produce the molecular jet used in the experiments, ${ }^{43,52}$ there is no ortho/para interconversion. Therefore the formula to determine the rate coefficients under these conditions is

$$
\begin{aligned}
k(T)= & w^{(o)} \frac{\int_{0}^{\infty} C_{r}^{(o)}(E) e^{-E / k_{B} T} d E}{h \Phi_{\mathrm{rel}}(T) Q_{v, j}^{o-\mathrm{H}_{2}}(T)} \\
& +w^{(p)} \frac{\int_{0}^{\infty} C_{r}^{(p)}(E) e^{-E / k_{B} T} d E}{h \Phi_{\mathrm{rel}}(T) Q_{v, j}^{p-\mathrm{H}_{2}}(T)},
\end{aligned}
$$

where $w^{(o)}$ and $w^{(p)}$ are the fractions of ortho- $\mathrm{H}_{2}$ and para- $\mathrm{H}_{2}$ at room temperature $\left(\approx 0.25\right.$ and 0.75 , respectively), $C_{r}^{(o)}(E)$ and $C_{r}^{(p)}(E)$ are the separate total CRPs for ortho and para, 
and $Q_{v, j}^{o-\mathrm{H}_{2}}(T), Q_{v, j}^{p-\mathrm{H}_{2}}(T)$ the corresponding partition functions for the two species.

For the purpose of this work, under the conditions just commented on above, it is also interesting to define the thermal-CRP including the reagent's partition function as

$$
\begin{aligned}
C_{r}(E ; T)= & w^{(o)} \frac{C_{r}^{(o)}(E) e^{-E / k_{B} T}}{h \Phi_{\mathrm{rel}}(T) Q_{v, j}^{o-\mathrm{H}_{2}}(T)} \\
& +w^{(p)} \frac{C_{r}^{(p)}(E) e^{-E / k_{B} T}}{h \Phi_{\mathrm{rel}}(T) Q_{v, j}^{p-\mathrm{H}_{2}}(T)},
\end{aligned}
$$

whose integration over the total energy range recovers the $k(T)$ given by Eq. (3). The thermal-CRP represents the contribution of the Boltzmann weighted CRP to the rate coefficient in an interval of energies within $E$ and $E+d E$.

For the QCT determination of the thermal rate coefficients there is no need to calculate the total CRP explicitly; the data of individual trajectories can be directly used as described in Ref. 13, allowing the incorporation of the Gaussian binning (GB) procedure. ${ }^{53,54}$ When this procedure is used, the trajectories are also weighted according to the proximity of their vibrational actions to those given by the correct (integer) QM vibrational quantum numbers. The weights are calculated using a Gaussian distribution function centered at the QM vibrational quantum numbers and with a full-width-halfmaximum of 0.1 .

\section{B. The quantum mechanical hyperspherical approach}

The hyperspherical quantum reactive scattering method developed by Launay and Dourneuf ${ }^{55}$ was described in detail in previous works. ${ }^{56}$ Recent modifications of the method, performed in order to allow the accurate inclusion of long-range interactions, were described in depth in Ref. 45 in the context of our study of the title reaction at low collision energies. Such modifications are not necessary in the present work. In what follows, we will simply recall the basic concepts while referring to previous works for more details.

According to this approach, the configuration space is divided into inner and outer regions. The position of the nuclei in the inner region is described in terms of hyperspherical democratic coordinates. The logarithmic derivative of the wavefunction is propagated outwards on a single adiabatic PES (the ground adiabatic PES in the current case). At a large enough value of the hyperradius the former is matched to a set of suitable functions, called asymptotic functions, to yield the scattering $S$-matrix. The asymptotic functions provide the collision boundary conditions and, when working at thermal energies, they are the familiar regular and irregular radial Bessel functions.

The $\boldsymbol{S}$ matrix for the title collision on the RKHS PES has been calculated for total angular momentum, $J=0,4$, and 10 on a dense grid of equally spaced energies. In addition, QM calculations for $J=0$ have been carried out on the DMBE/SEC PES. Total energies ranging from the opening of the ground state of $\mathrm{H}_{2}(E \approx 0.27 \mathrm{eV})$ up to $1 \mathrm{eV}$ were considered. Calculations have been also performed for $J=30$ in the same energy range but only at a reduced number of total ener- gies, given the rapidly growing computational effort required as the total angular momentum increases. The convergence parameters used in the current work are the same as those used in the study of the title collision at thermal energies, and are given in Refs. 32 and 36. In particular, note that an intermolecular separation of $\sim 10 \mathrm{a}_{0}$ was chosen for the matching of the inner propagation with the asymptotic functions.

\section{Statistical quasi-classical trajectory calculations}

The statistical quasi-classical trajectory method (SQCT) has been presented in detail in previous publications. ${ }^{19,20}$ The determination of the SQCT CRP is described in Ref. 18. The SQCT results for the reaction under study on the RKHS PES were found to be in almost perfect agreement with those obtained using the quantal version (SQM) ${ }^{35}$

The SQCT CRPs were obtained for the same total angular momentum values and PES as in the QCT case. Batches of $10^{6}$ trajectories were run for each arrangement. The starting intermolecular distance was set to $10 \AA$ ( $30 \AA$ at the lowest total energies) and the integration step was $0.08 \mathrm{fs}$, small enough to ensure an energy conservation better than 1 part in $10^{5}$. The values of the capture potential were set at $-0.6 \mathrm{eV}$ and $-0.8 \mathrm{eV}$ for the reactant and product channels, respectively. ${ }^{35}$

In addition, calculations at 35 fixed total energies, in the $0.27-1.3 \mathrm{eV}$ total energy range, were calculated to determine the total CRP and thermal rate coefficients on both PESs using the equations of Subsection II A.

\section{RESULTS}

\section{A. Cumulative reaction probability for $J=0$}

The cumulative reaction probabilities for $J=0$, obtained using the three different approaches, are shown in the top and bottom panels of Fig. 1 for the DMBE/SEC and RKHS PESs, respectively. Unless stated on the contrary, the CRPs that will be shown are the unweighted sum of the contributions for para- $\mathrm{H}_{2}\left(j\right.$ even) and ortho $-\mathrm{H}_{2}$ ( $j$ odd). In the case of the QM results, the underlying step-like structure is in part obscured by the resonance pattern. Except for the resonance oscillations, the QCT results are in good agreement with the QM ones over the considered energy range. In the QCT and SQCT CRP calculations the step-like structure is more clearly shown, reflecting the opening of the reactant states. In contrast to the $J=0 \mathrm{CRP}$ for the $\mathrm{H}^{+}+\mathrm{H}_{2}$ system, ${ }^{18}$ the opening of the product states does not increase the reactivity. Although the $\mathrm{S}\left({ }^{1} D\right)+\mathrm{H}_{2}$ is only mildly exoergic, at the time of the opening of the lowest $\mathrm{H}_{2}$ state there are already about 20 rovibrational states available for the $\mathrm{SH}$ product and therefore the addition of successive higher product states does not increase the reactive flux significantly. As can be seen, the statistical model overestimates the reactivity with respect to the QM or QCT results in the energy range considered. The calculations on the RKHS PES (bottom panel of Fig. 1) lead to a substantially better agreement between the SQCT and QM results, especially at the highest energies. This indicates 

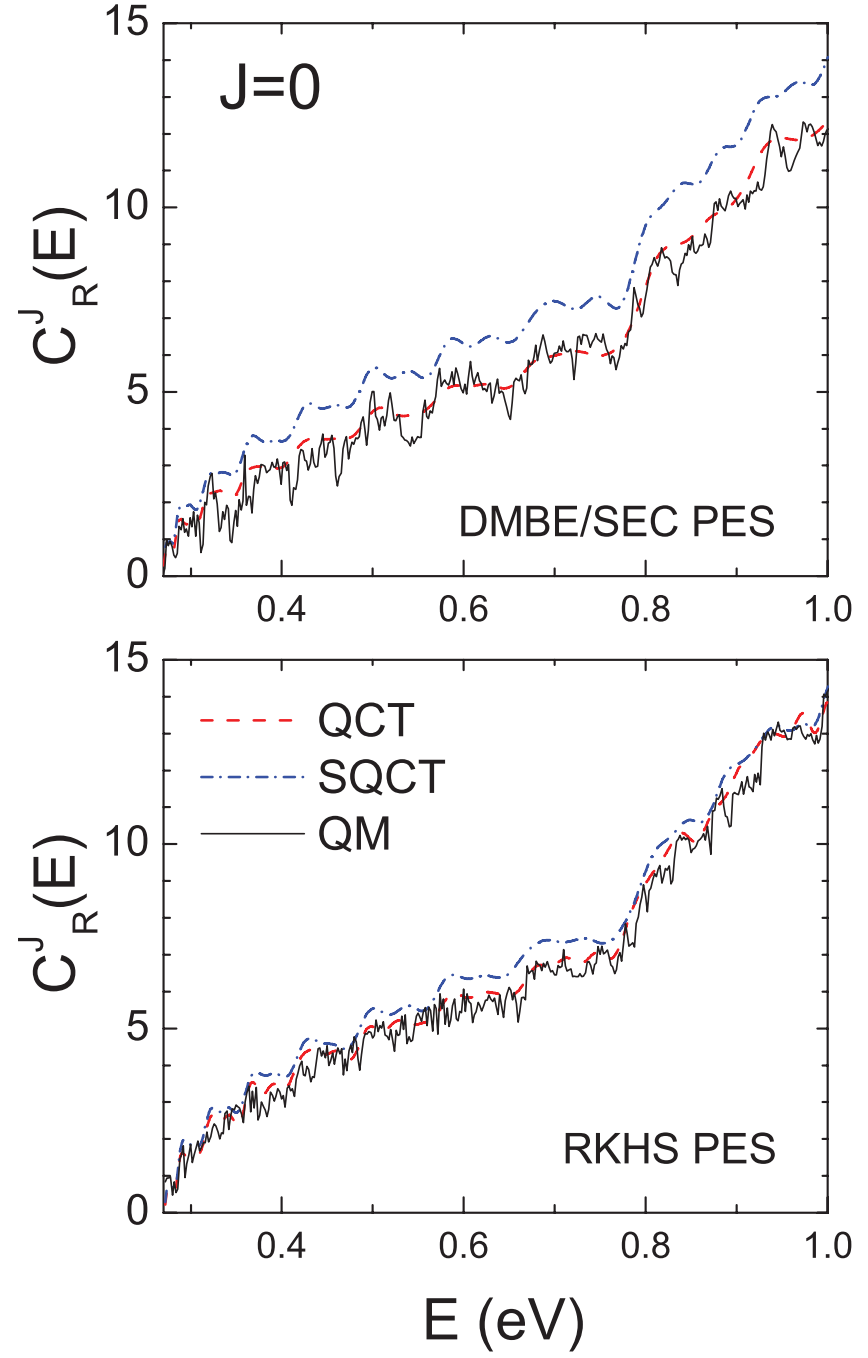

FIG. 1. Cumulative Reaction Probability for $J=0, C_{R}^{J=0}(E)$, on the DMBE/SEC PES (upper panel) and the RKHS PES (lower panel). Solid black line: QM results. Dashed red line: QCT results. Dashed-dotted blue line: SQCT results.

that the behavior on the RKHS PES is more statistical than that observed on the DMBE/SEC PES.

A deeper insight into the origin of the discrepancies between the calculations on both PESs is achieved from Fig. 2, where the results are separately compared for each theoretical method. The top panel, where the QM CRPs are shown for the two PESs, indicates that the reactivity on the RKHS PES is slightly larger. Interestingly, the step-like pattern in the CRP becomes more evident for $v=1$ on the RKHS PES. The fact that observed differences between the CRPs on the two PESs also appear in the QCT results (middle panel) seems to rule out a purely quantal origin of those differences. Actually, the absence of the resonance structure makes more evident the extent of the discrepancies between the results on the two PES. Finally, the bottom panel of Fig. 2 shows that the SQCT CRPs on the RKHS and DMBE/SEC PESs (bottom panel) are almost indistinguishable except at the lowest energies. Since the statistical calculations only consider the entrance and exit valleys (up to the capture point), it can be concluded that most of the differences must be attributed to
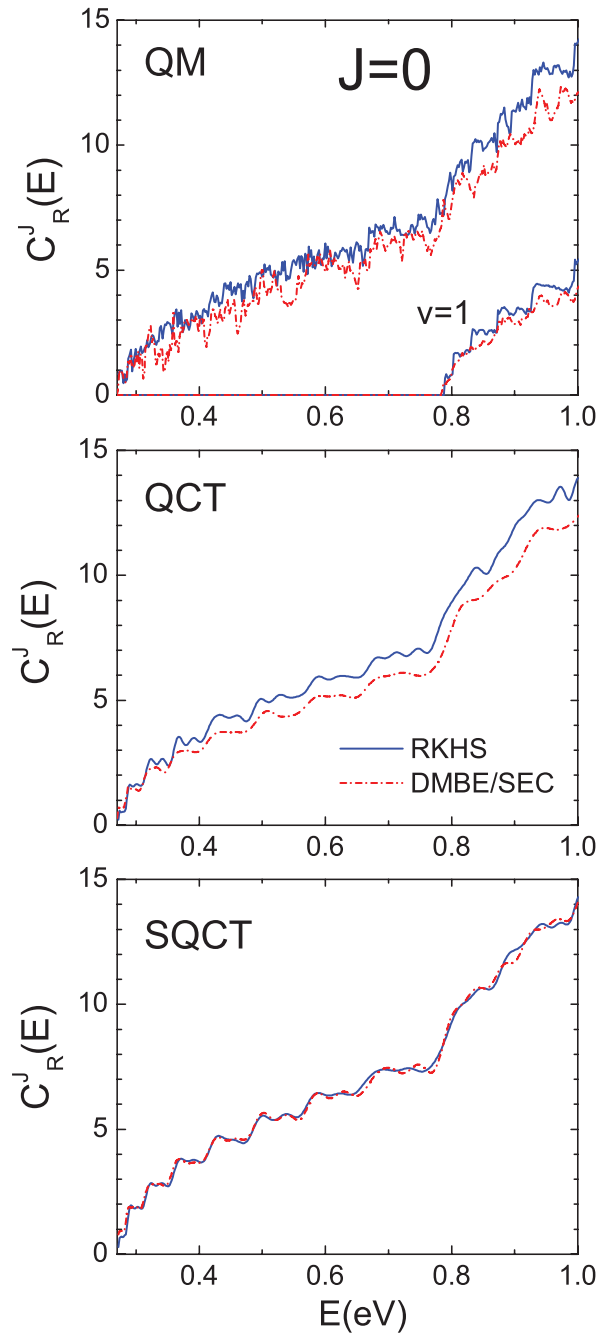

FIG. 2. Comparison between the CRPs for $J=0$ obtained with the three theoretical approaches on the indicated PESs (solid blue line: RKHS; dasheddotted red line: DMBE/SEC). Upper panel: QM results; middle panel: QCT results; lower panel: SQCT results. The QM CRP for $v=1$ is also displayed.

the topography of the well that is disregarded in the statistical approximations.

Had we determined the CRPs on the DMBE/CBS PES, ${ }^{46}$ we would have found noteworthy differences with the results obtained on the RKHS and DMBE/SEC PES. This is due to the fit resulting in DMBE/CBS PES that produced some artifacts which amounts to a barrier at compressed $\mathrm{H}-\mathrm{H}$ internuclear distances. ${ }^{49}$ To highlight these differences Fig. 3 shows the reaction probability for $J=0$ corresponding to two different initial vibrational states $v=0, j=0$ and $v=1$, $j=0$. Results on the three PESs, RKHS, DMBE/CBS, and DMBE/SEC, are shown in the left, middle, and right panels of Fig. 3, respectively. While differences among the results on the three PES for $(v=0, j=0)$ are relatively minor, especially at high collision energies, the results for $(v=1$, $j=0$ ) reveal the effect of the artificial barrier on the DMBE/CBS PES as the appearance of an energy threshold of $\sim 25 \mathrm{meV}$, which is observed regardless of the theoretical method employed. Interestingly, the QM and QCT reactivities are larger on the DMBE/CBS PES than on the DMBE/SEC PES for energies well above the spurious $v=1$ threshold. 

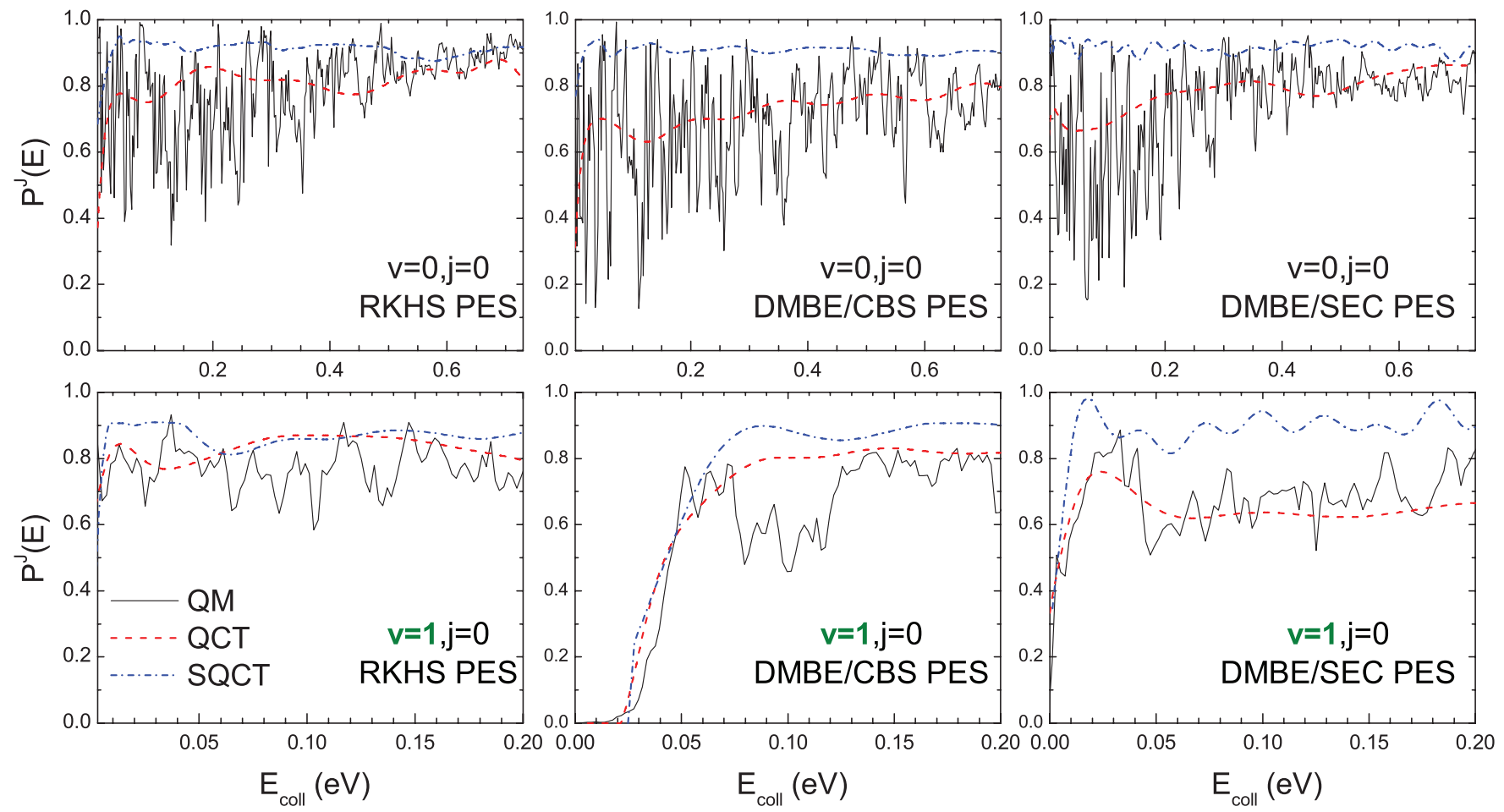

FIG. 3. Reaction probability for $J=0$ as a function of the collision energy for $(v=0, j=0)$ (upper panels) and $(v=1, j=0)$ (lower panels) initial states, calculated on three different PES (RKHS: left panel; DMBE/CBS: ${ }^{46}$ middle panel; DMBE/SEC: right panel). On the DMBE/CBS PES there is a threshold of $\approx 25 \mathrm{meV}$ for $v=1$. Such threshold does not appear on the two other PESs.

\section{B. Cumulative reaction probabilities for $J \neq 0$}

The CRPs for fixed $J$ greater than zero have been also calculated on the RKHS PES. In Fig. 4 we display the results for $J=4,10,30$, and 50 , which were obtained by using
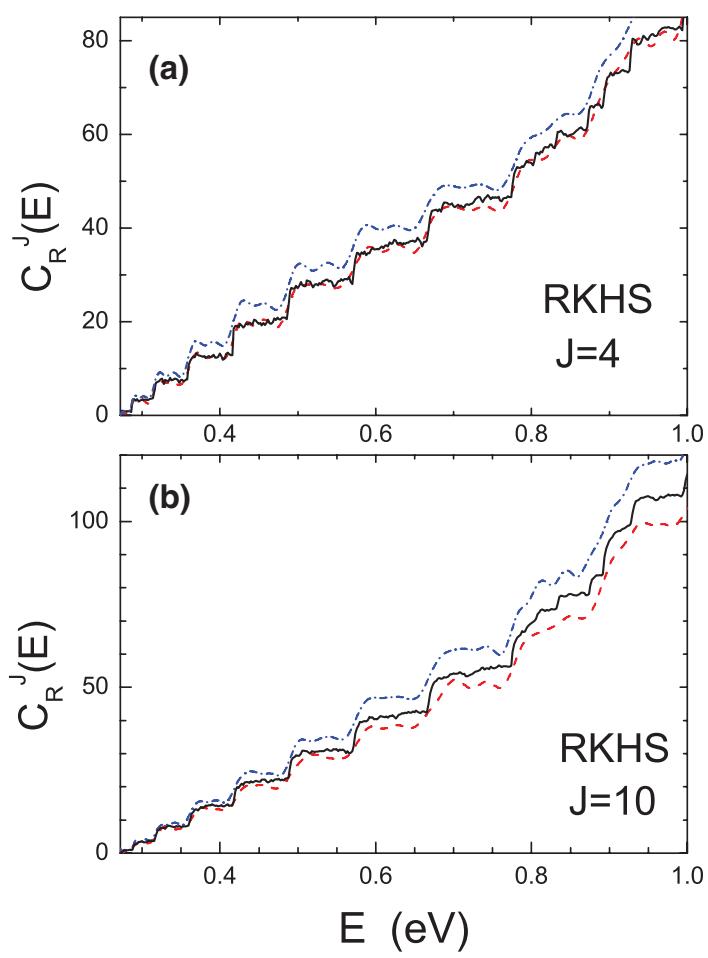

the three different methods. On panel (a), the step-like structure in QM CRP for $J=4$ becomes apparent. QCT results show a nearly perfect agreement with the $\mathrm{QM}$ ones whereas the SQCT method overestimates the reactivity. For $J=10$,
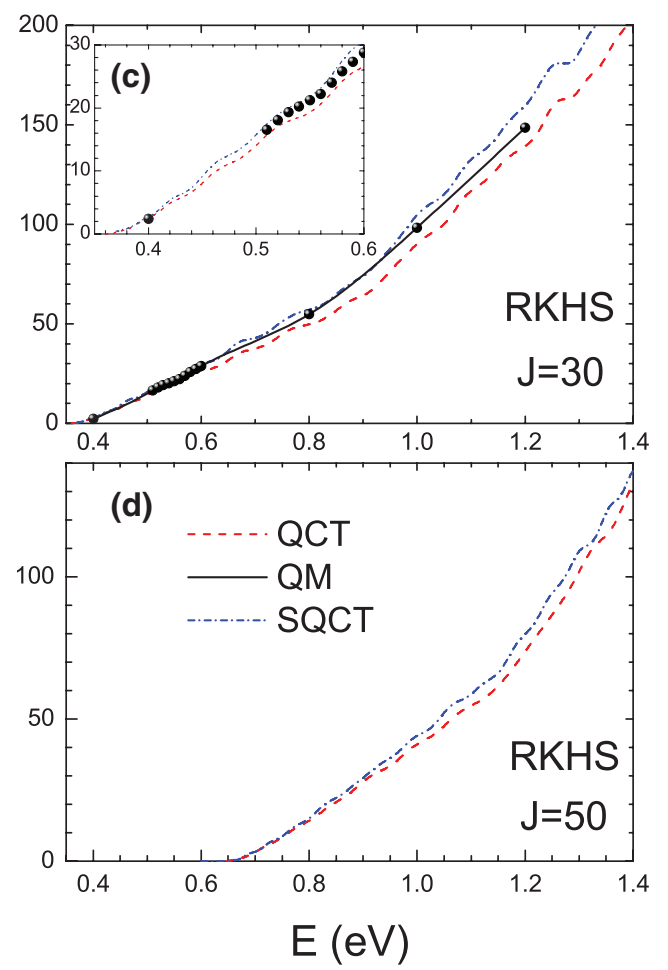

FIG. 4. Cumulative reaction probabilities as a function of the total energy for $J=4,10,30$, and 50 calculated on the RKHS PES. Solid black line: QM results. Dashed red line: QCT results. Dashed-dotted blue line: SQCT. Only a reduced set of energies have been calculated for $J=30$ using the QM approach. 
on panel (b), the step structure is still visible and QCT results follow the same trend as the QM ones, although the agreement is slightly worse, especially at higher energies. It must be stressed that the QM calculations have been carried out without resorting to any approximation.

Results for $J=30$ are displayed on panel (c). Only few representative energies were considered in the QM calculations due to the huge computational cost when the full Coriolis coupling is included. At this relatively large value of $J$, the centrifugal barrier dominates the reactivity and the steplike structure disappears. Besides, the extent of the difference between the QCT and SQCT results is similar to that found for lower total angular momenta, what actually means that the statistical behavior does not disappear at high energies. It must be pointed out that at these high $J$ values the QM CRPs are in somewhat better agreement with the SQCT CRPs than with those obtained with the QCT method. Finally, QCT and SQCT results for $J=50$ are very much alike (panel (d)). Therefore, QM CRPs for $J>30$, not calculated because of their computational cost, are expected to be in good accordance with those from the QCT and SQCT approaches. These results are in strong contrast with those obtained for the $\mathrm{H}^{+}+\mathrm{H}_{2}$ reaction and isotopic variants, for which the differences between SQCT and QCT results for sufficiently large $J$ values and energies were remarkable. ${ }^{18}$

\section{Total CRPs and thermal rate coefficients}

Once the CRPs calculated with the QCT, SQCT, and QM approaches have been compared using the different PESs here considered, our attention focus on the determination of the thermal rate coefficients, $k(T)$.

As indicated in Subsection II A, thermal rate coefficients have been calculated from the total CRP, $C_{R}(E)$. The comparison of the total CRP calculated on the RKHS and DMBE/SEC PESs using the QCT and SQCT methods are shown in Fig. 5. The inset in this figure shows an enlargement of the low en-

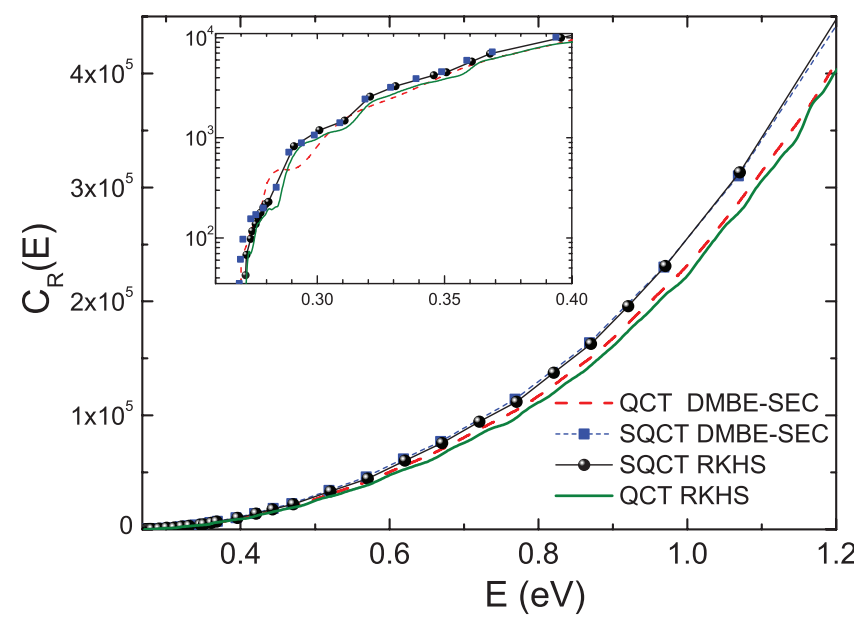

FIG. 5. Total CRP (comprising $\mathrm{H}_{2}$ even and odd rotational states according to Eq. (2)) as a function of the total energy. Lines and symbols as indicated in the figure. SQCT results were calculated at several fixed energies and are shown as circles and squares for the RKHS and DMBE/SEC PESs, respectively.
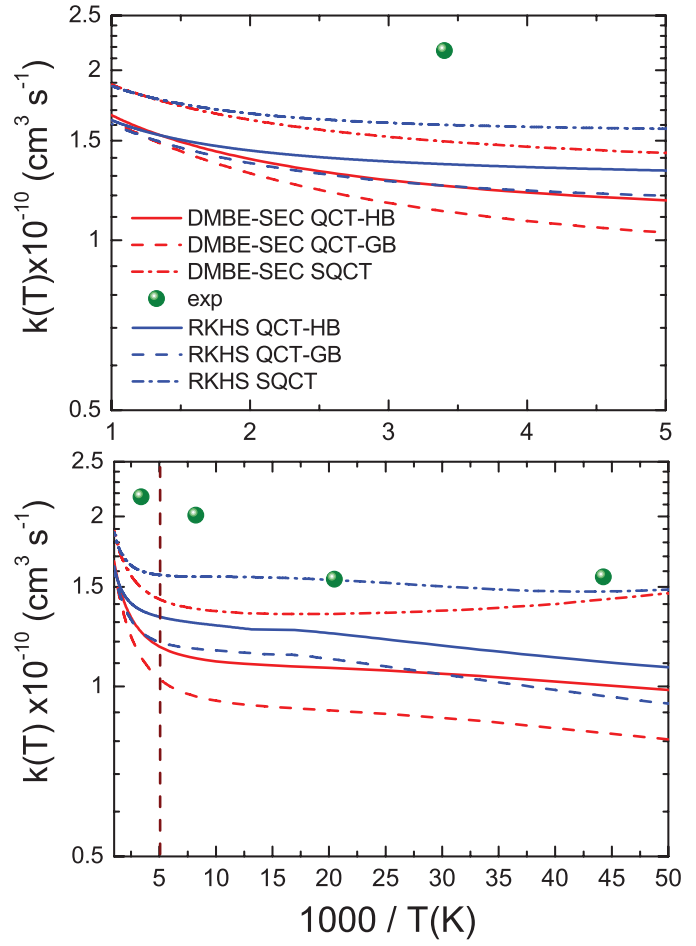

FIG. 6. Arrhenius plot of the QCT-HB, QCT-GB, and SQCT thermal rate coefficients, $k(T)$, calculated on the RKHS (blue lines) and DMBE/SEC (red lines). The bottom panel comprises temperatures from $20-1000 \mathrm{~K}$. The region to the left of the vertical dashed line $(200-1000 \mathrm{~K})$ in the bottom panel is expanded in the upper panel. The experimental data appear as green solid points. $^{43}$

ergy region in logarithmic scale. Since the QM CRPs were calculated only for a very limited number of total angular momenta, the QM total CRP is not available. However, the good accordance of the QM results with the SQCT and the QCT data lends credence to the calculations using these approaches. As shown in Fig. 5, the differences between the total CRPs on both PESs are relatively minor in most of the energy range, although there are some differences at low energies $(0.27-0.35 \mathrm{eV})$. The SQCT CRPs are slightly larger than those obtained with the QCT method in the whole range of total energies considered in this work, and the relative difference between QCT and SQCT data does not increase with the energy.

Arrhenius plots of the thermal rate coefficients calculated from the total CRPs (see Subsection II A), are shown in Fig. 6. The results using the standard (histogram) binning, QCT-HB, the Gaussian binning, QCT-GB, and the SQCT methods are depicted in the figure. The top panel of the figure shows the results covering the range 200-1000 K, while the bottom panel of Fig. 6 extends the range of temperatures down to $20 \mathrm{~K}$. Irrespective of the calculation method, the rate coefficients obtained using the RKHS PES are systematically larger than those obtained on the DMBE/PES. Only at the highest temperatures $(\approx 500 \mathrm{~K})$, their values seem to converge. The similarity of the results on both surfaces at the highest temperatures is not surprising as the total CRPs become very similar with increasing energy. The $k(T)$ values predicted by the SQCT model are closer to the experimental data by Sims and co-workers ${ }^{43}$ which are also shown in the 

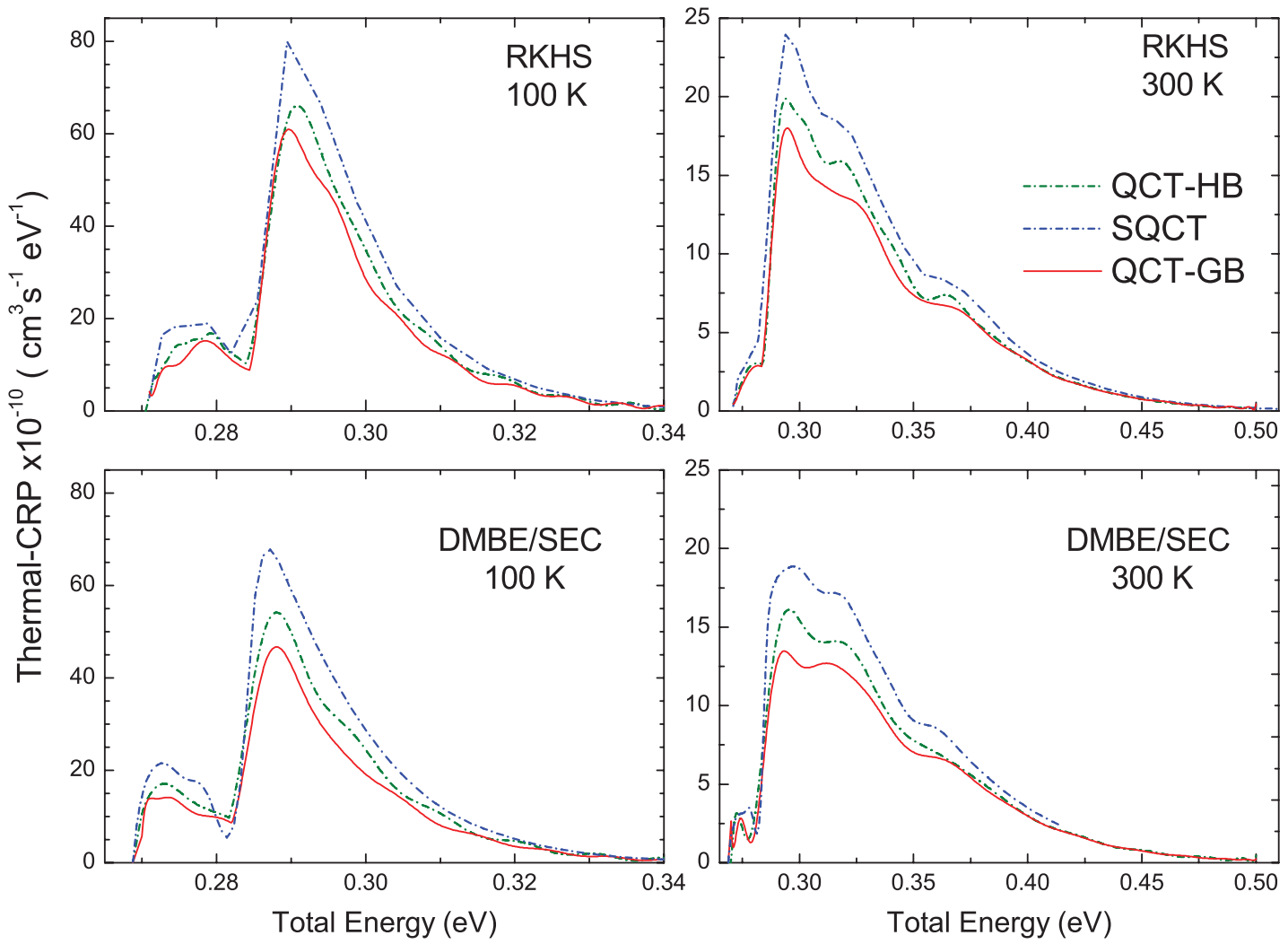

FIG. 7. Thermal cumulative reaction probabilities divided by the partition function as a function of total energy (such that the integral of each curve gives the corresponding rate coefficient) at $T=100 \mathrm{~K}$ (left panels) and $300 \mathrm{~K}$ (right panels). Top row: calculations on the RKHS PES. Bottom row: on the DMBE/SEC PES. The first peak is due to the $j=0$ contribution, while the second contains the contributions of $j=0$ and $j=1$ taking into account the $p$ - $\mathrm{H}_{2}$ and $o$ - $\mathrm{H}_{2}$ abundances $(\approx 25 \%$ and $\approx 75 \%$, respectively).

figure. It is important to notice that the experimental $k(T)$ corresponds to the total removal of $\mathrm{S}\left({ }^{1} D\right)$ atoms, thus accounting for both the reaction and the electronic quenching to $S\left({ }^{3} P\right)$. The experimental data constitute an upper limit to the actual reaction rate coefficients and, as discussed in Ref. 45, are expected to be somewhat higher than the theoretical values obtained in an adiabatic treatment. Multistate non-adiabatic calculations ${ }^{40}$ including the triplet PES and the spin-orbit couplings for the $\mathrm{S}\left({ }^{1} D\right)+\mathrm{HD}$ reaction indicate that at least for this isotopic variant non-adiabatic effects caused by intersystem crossing are important and that the electronic quenching is comparable with the reactive cross section.

To gain more information about the differences in the values of the rate coefficients, thermal cumulative reaction probabilities (or thermal-CRP) have been calculated at $100 \mathrm{~K}$ and $300 \mathrm{~K}$, and are shown in Fig. 7 in the left and right panels, respectively. The thermal-CRP corresponding to the RKHS PES and $100 \mathrm{~K}$ (top left panel) exhibits a shoulder associated to the contribution of $j=0$, followed by a maximum at about $290 \mathrm{meV}$, which is due to the contribution of $j=1$ (recall that odd $j$ states have larger weights). After this maximum, the thermal CRP decreases following the Boltzmann factor. The curves at $300 \mathrm{~K}$ (right panels) are much broader (note the different energy scale) and the successive contributions from the opening of higher $\mathrm{H}_{2}$ rovibrational states appear as superimposed shoulders as the energy increases. The curves resulting from the various approaches display the same trend, although the SQCT results are always larger than the QCT ones in the whole energy range, and this is especially noticeable in the height of the maxima. As it could be anticipated, differences between both QCT methods disappear as the energy increases.

The thermal-CRPs calculated on the DMBE/SEC PES (bottom panels) are similar to those found using the RKHS PES, following basically the same pattern although the magnitude of the thermal-CRPs on the latter is clearly bigger, what is reflected in the larger values of the corresponding $k(T)$. However, at $100 \mathrm{~K}$ the relative contribution of $j=0$ with respect to that from $j=1$ is somewhat more important in the case of the DMBE/SEC PES.

\section{CONCLUSIONS}

In this work we have performed a detailed analysis of the $\mathrm{S}\left({ }^{1} D\right)+\mathrm{H}_{2}$ reaction using the cumulative reaction probability (CRP) methodology. Calculations of CRPs at various total angular momenta have been carried out using the timeindependent quantum mechanical (QM), quasiclassical trajectories (QCT), and the statistical quasiclassical trajectory (SQCT) model approaches. Two potential energy surfaces (PESs) were considered: the widely used RKHS and the recent DMBE/SEC PESs. In addition, total CRPs and thermal rate coefficients in the $20-1000 \mathrm{~K}$ have been calculated using the QCT and SQCT methods.

For this barrierless reaction, the dynamical bottleneck is shifted towards the reagent asymptote for low $J$ values and 
the CRP shows a step-like structure even for $J=0$ (although partially blurred by the resonance pattern in the QM calculations). These steps reflect the opening and reactivity of the initial states of the $\mathrm{H}_{2}$ reagent. For larger $J$ values, the effect of the centrifugal barrier damps the step-like structure. The CRPs calculated with the three different theoretical methodologies are in very good agreement even for the largest total angular momentum calculated. Only at low $J$ values, the SQCT CRPs tend to overestimate the QM results slightly.

The total SQCT CRPs and hence the rate coefficients are larger than those found using the QCT approach, leading to a somewhat better agreement with the existing experimental data. Caution should be exercised in this comparison since the experimental data corresponds to total - reaction plus electronic quenching - removal rates of $S\left({ }^{1} D\right)$. As for the comparison between the results on the two PESs here scrutinized, it can be concluded that the existing differences are relatively small and can be traced back to the differences in the topology of the well. In fact, except at the lowest energies, the SQCT CRPs calculated on both PESs are almost identical. In contrast, differences can be appreciated in the QM as well as in the QCT results, what rules out their attribution to pure quantum effects. The rate coefficients obtained on the RKHS PES are slightly larger on the whole range of temperatures (no more than 15\%) but tend to converge at the highest temperatures.

As a whole, taking into account all the existing dynamical information, the reaction can be considered as statistical, dynamically dominated by the capture before the well and hence quantitatively describable by QM or QCT statistical models even in the high energy regime. This is in contrast to other prototypical reactions, such as $\mathrm{H}^{+}+\mathrm{H}_{2}$, long considered statistical but whose behavior departs from that category as the energy increases.

\section{ACKNOWLEDGMENTS}

The authors acknowledge funding by the Spanish Ministry of Science and Innovation (Grant Nos. CTQ2008-02578 and Consolider Ingenio 2010 CSD2009-00038).

${ }^{1}$ W. H. Miller, J. Chem. Phys. 62, 1899 (1975).

${ }^{2}$ W. H. Miller, J. Chem. Phys. 65, 2216 (1976).

${ }^{3}$ W. H. Miller, Acc. Chem. Res 9, 306 (1976).

${ }^{4}$ W. H. Miller, Acc. Chem. Res 26, 174 (1993).

${ }^{5}$ D. C. Chatfield, R. S. Friedman, D. G. Truhlar, B. C. Garrett, and D. W. Schwenke, J. Am. Chem. Soc. 113, 486 (1991).

${ }^{6}$ D. C. Chatfield, R. S. Friedman, D. G. Truhlar, and D. W. Schwenke, Faraday Discuss. Chem. Soc. 91, 289 (1991).

${ }^{7}$ D. C. Chatfield, R. S. Friedman, D. W. Schwenke, and D. G. Truhlar, J. Phys. Chem. 96, 2414 (1992).

${ }^{8}$ D. Dai, C. C. Wang, S. A. Harich, X. Wang, X. Yang, S. D. Chao, and R. T. Skodje, Science 300, 1730 (2003).

${ }^{9}$ H. Eyring, J. Chem. Phys. 3, 107 (1935)

${ }^{10} \mathrm{H}$. Eyring and M. Polanyi, Trans. Faraday Soc. 31, 875 (1935).

${ }^{11}$ D. R. Herschbach, Faraday Discuss. Chem. Soc. 55, 233 (1975).

${ }^{12}$ W. H. Miller, S. A. Safron, and D. R. Herschbach, Discuss. Faraday Soc. 44, 108 (1967)

${ }^{13}$ F. J. Aoiz, M. Brouard, C. J. Eyles, J. F. Castillo, and V. Sáez Rábanos, J. Chem. Phys. 125, 144105 (2006).

${ }^{14}$ Y. Lin, B. Ramachandran, K. Nobusada, and H. Nakamura, J. Chem. Phys. 116, 7461 (2000).
${ }^{15}$ Y. Lin, B. Ramachandran, K. Nobusada, and H. Nakamura, J. Chem. Phys. 114, 1549 (2001).

${ }^{16}$ F. J. Aoiz, V. J. Herrero, M. P. de Miranda, and V. Sáez Rábanos, Phys. Chem. Chem. Phys. 9, 5367 (2007).

${ }^{17}$ F. J. Aoiz, V. J. Herrero, and V. Sáez Rábanos, J. Chem. Phys. 129, 024305 (2008).

${ }^{18}$ P. G. Jambrina, F. J. Aoiz, C. J. Eyles, V. J. Herrero, and V. Sáez-Rábanos, J. Chem. Phys. 130, 184303 (2009).

${ }^{19}$ F. J. Aoiz, V. Sáez-Rábanos, T. González-Lezana, and D. E. Manolopoulos, J. Chem. Phys. 126, 161101 (2007).

${ }^{20}$ F. J. Aoiz, T. González-Lezana, and V. Sáez-Rábanos, J. Chem. Phys. 127, 174109 (2007)

${ }^{21}$ P. G. Jambrina, F. J. Aoiz, J. M. Alvariño, V. J. Herrero, and V. SáezRábanos, Phys. Chem. Chem. Phys. 12, 12591 (2010).

${ }^{22}$ P. G. Jambrina, F. J. Aoiz, N. Bulut, S. C. Smith, G. G. Balint-Kurti, and M. Hankel, Phys. Chem. Chem. Phys. 12, 1102 (2010).

${ }^{23}$ P. G. Jambrina, J. M. Alvariño, D. Gerlich, M. Hankel, V. J. Herrero, V. Sáez-Rábanos, and F. J. Aoiz, Phys. Chem. Chem. Phys. 14, 3346 (2012).

${ }^{24}$ Y. Inagaki, S. M. Shamsuddin, Y. Matsumi, and M. Kawasaki, Laser Chem. 14, 235 (1994)

${ }^{25}$ S.-H. Lee and K. Liu, Chem. Phys. Lett. 290, 323 (1998).

${ }^{26}$ S.-H. Lee and K. Liu, J. Phys. Chem. A 102, 8637 (1998).

${ }^{27}$ S.-H. Lee and K. Liu, Appl. Phys. B: Lasers Opt. 71, 627 (2000).

${ }^{28}$ F. J. Aoiz, L. Bañares, and V. J. Herrero, J. Phys. Chem. A 110, 12546 (2006).

${ }^{29}$ A. S. Zyubin, A. M. Mebel, S. D. Chao, and R. T. Skodje, J. Chem. Phys. 114, 320 (2001).

${ }^{30}$ T. S. Ho, T. Hollebeek, H. Rabitz, S. C. Chao, R. T. Skodje, A. S. Zyubin, and A. M. Mebel, J. Chem. Phys. 116, 4124 (2002).

${ }^{31}$ S. D. Chao and R. T. Skodje, J. Phys. Chem. A 105, 2474 (2001).

${ }^{32}$ L. Bañares, J. F. Castillo, P. Honvault, and J.-M. Launay, Phys. Chem. Chem. Phys. 7, 627 (2005).

${ }^{33}$ B. Maiti, G. C. Schatz, and G. Lendvay, J. Phys. Chem. A 108, 8772 (2004).

${ }^{34}$ P. Honvault and J.-M. Launay, Chem. Phys. Lett. 370, 371 (2003).

${ }^{35}$ F. J. Aoiz, T. González-Lezana, and V. Sáez-Rábanos, J. Chem. Phys. 129, 094305 (2008).

${ }^{36}$ L. Bañares, F. J. Aoiz, P. Honvault, and J.-M. Launay, J. Phys. Chem. A 108, 1616 (2004).

${ }^{37}$ E. J. Rackham, T. González-Lezana, and D. E. Manolopoulos, J. Chem. Phys. 119, 12895 (2003).

${ }^{38}$ S. Y. Lin and H. Guo, J. Chem. Phys. 122, 074304 (2005).

${ }^{39}$ J. A. Kłos, P. J. Dagdigian, and M. H. Alexander, J. Chem. Phys. 127, 154321 (2007).

${ }^{40}$ T.-S. Chu, K.-L. Han, and G. C. Schatz, J. Phys. Chem. A 111, 8286 (2007).

${ }^{41}$ H. Yang, K.-L. Han, G. C. Schatz, S.-H. Lee, K. Liu, S. C. Smith, and M. Hankel, Phys. Chem. Chem. Phys. 11, 11587 (2009).

${ }^{42}$ H. Guo, Int. Rev. Phys. Chem. 31, 1 (2012).

${ }^{43}$ C. Berteloite, M. Lara, A. Bergeat, S. D. Le Picard, F. Dayou, K. M. Hickson, A. Canosa, C. Naulin, J. M. Launay, I. R. Sims et al., Phys. Rev. Lett. 105, 203201 (2010).

${ }^{44}$ M. Lara, F. Dayou, J. M. Launay, A. Bergeat, K. M. Hickson, C. Naulin, and M. Costes, Phys. Chem. Chem. Phys. 13, 8127 (2011).

${ }^{45}$ M. Lara, F. Dayou, and J. M. Launay, Phys. Chem. Chem. Phys. 13, 8359 (2011).

${ }^{46}$ Y. Z. Song and A. J. C. Varandas, J. Chem. Phys. 130, 134317 (2009).

${ }^{47}$ Y. Z. Song, P. J. S. B. Caridade, and A. J. C. Varandas, J. Phys. Chem. A 113, 9213 (2009).

${ }^{48}$ C. Schlier and U. Vix, Chem. Phys. 113, 211 (1987).

${ }^{49}$ M. Lara, P. G. Jambrina, A. J. C. Varandas, J. Launay, and F. J. Aoiz, J. Chem. Phys. 135, 134313 (2011).

${ }^{50}$ M. Hankel, S. C. Smith, and A. J. C. Varandas, Phys. Chem. Chem. Phys. 13, 13645 (2011).

${ }^{51}$ F. J. Aoiz, V. Sáez-Rábanos, B. Martínez-Haya, and T. González-Lezana, J. Chem. Phys. 123, 094101 (2005).

${ }^{52}$ A. Canosa, F. Goulay, I. R. Sims, and B. R. Rowe, Low Temperatures and Cold Molecules (World Scientific, Singapore, 2008).

${ }^{53}$ L. Bonnet and J. C. Rayez, Chem. Phys. Lett. 397, 106 (2004).

${ }^{54}$ L. Bañares, F. J. Aoiz, P. Honvault, B. Bussery-Honvault, and J. M. Launay, J. Chem. Phys. 118, 565 (2003).

${ }^{55}$ J. M. Launay and M. L. Dourneuf, Chem. Phys. Lett. 169, 473 (1990).

${ }^{56}$ P. Honvault and J.-M. Launay, in Theory of Chemical Reaction Dynamics, NATO Science Series Vol. 145 (Kluwer, 2004). 